\title{
IGF2BP2 Promotes Pancreatic Carcinoma \\ Progression by Enhancing the Stability of B3GNT6 mRNA via m6A Methylation
}

\section{Xiaofeng Xue ( $\nabla$ xfxue@suda.edu.cn )}

First Affiliated Hospital of Soochow University https://orcid.org/0000-0002-0963-7994

\section{Pei Cao}

First Affiliated Hospital of Soochow University Department of General Surgery

\section{Yufan Wu}

First Affiliated Hospital of Soochow University Department of General Surgery

\section{Ding Sun}

First Affiliated Hospital of Soochow University Department of General Surgery

\section{Weigang Zhang}

First Affiliated Hospital of Soochow University Department of General Surgery

\section{Junyi Qiu}

First Affiliated Hospital of Soochow University Department of General Surgery

\section{Zuxiong Tang}

First Affiliated Hospital of Soochow University Department of General Surgery

\section{Lei Qin}

First Affiliated Hospital of Soochow University Department of General Surgery

\section{Research}

Keywords: Pancreatic carcinoma, m6A methylation, IGF2BP2, B3GNT6

Posted Date: August 30th, 2021

DOI: https://doi.org/10.21203/rs.3.rs-805683/v1

License: (1) (1) This work is licensed under a Creative Commons Attribution 4.0 International License. Read Full License 


\section{Abstract}

\section{Background}

Pancreatic carcinoma (PC) is a highly lethal cancer with an increasing mortality rate, its five-year survival rate is only approximately $4 \%$. N6-methyladenosine (m6A) modification is the most common posttranscriptional modification of RNA. However, its role in PC remains unclear.

\section{Methods}

We combined bioinformatic analysis with in vitro and in vivo experiments to investigate the expression profile of methylation modulators and identify key m6A regulators in the progression of PC. Further study focused on exploring the target genes binding to the regulators through RIP and double fluorescence staining.

\section{Results}

TCGA and Gene Expression Omnibus (GEO) analyses revealed an overall increasing trend in the expression of m6A regulators in $\mathrm{PC}$, and consensus clustering analysis of m6A showed that the expression of regulators was negatively correlated with the survival rate. LASSO-Cox regression analysis revealed that IGF2BP2, METTL3, ALKBH5 and KIAA1429 were associated with hazard ratios (HR), but only IGF2BP2 was sufficiently appropriate for the m6A survival prognosis model. The IHC and WB results verified high protein expression of IGF2BP2 in PC, and IGF2BP2 knockdown inhibited the proliferation and migration of PC cells. We predicted nine possible target mRNAs of IGF2BP2, and B3GNT6, which regulates three-dimensional serine or threonine structures to affect biosynthesis and metabolism, was verified via RIP and dual luciferase reporter assays to be observably methylated by IGF2BP2. In addition, IF staining confirmed the co-expression of IGF2BP2 and B3GNT6. The tumour-promoting effect of IGF2BP2 and its co-expression with B3GNT6 were verified in an animal model.

\section{Conclusions:}

Elevated m6A levels promote PC progression. IGF2BP2 is a credible marker and modulates B3GNT6 mRNA stability, indicating that IGF2BP2 is a potential prognostic marker and therapeutic target in PC progression.

\section{Background}

PC has long been one of the most devastating and highly malignant tumours, with obvious resistance to radiotherapy and chemotherapy, the five-year survival rate is only $4 \%^{1}$. In both the United States and China, the morbidity and mortality of PC are among the highest of all cancers ${ }^{2}$. Currently, the most common and effective treatment is surgery combined with radiochemotherapy and immunotherapy, but this regimen exhibits little therapeutic effect ${ }^{3}$. Considering that PC is characterized by a strong interstitial 
hyperplastic reaction around cancer cells ${ }^{4}$, its drug resistance and early invasive metastasis control the high mortality of $\mathrm{PC}$ patients ${ }^{5}$. During the progression of $\mathrm{PC}$, different signalling pathways mediate cancer typing, growth, proliferation and invasion, which makes the tumour microenvironment more complex ${ }^{6}$. Given that the current treatment is not satisfactory, there is an urgent need to identify novel and unique approaches to improve the dismal prognosis of PC.

It is well known that RNA is subject to a variety of internal modifications, among which N6methyladenosine (m6A) methylation is the most abundant and conserved epigenetic chemical modification ${ }^{7}$, especially on eukaryotic messenger RNA (mRNA). Scientists have discovered that m6A methylation is a dynamic reversible process mediated by m6A methylation-related proteins ${ }^{8} . \mathrm{m} 6 \mathrm{~A}$ methyltransferase "writers" (METTL3/14, WTAP, RBM15 and KIAA1429) facilitate the installation of methyl groups on RNA, demethylation is mediated by "erasers" (FTO and ALKBH5), and methyl groups are recognized by "readers" (YTHDF1/2/3, IGF2BP1/2/3 and YTHDC1/2) to perform related functions", including the regulation of $m R N A$ stability ${ }^{10}$, transcription and translation efficiency ${ }^{11}$, RNA processing events ${ }^{12}$, miRNA maturation, IncRNA and circRNA polypeptide translation. It has been found that m6A mRNA methylation plays an essential role in the occurrence and development of tumours. In primary glioblastoma, m6A levels are significantly reduced compared with those in normal tissues. When GSCs are induced to differentiate, the m6A level increases, while inhibition of METTL3 or METTL14 expression can decrease the m6A level and enhance the tumorigenic ability of GSCs ${ }^{13}$; METTL3 increases the expression of the target genes C-MYC, BCL-2 and PTEN in AML to inhibit cell differentiation and apoptosis to promote cell proliferation ${ }^{14}$. Chen et al. found that METTL3 reduces the stability of SOCS2 mRNA through an m6A-YTHDF2-dependent pathway to promote the occurrence and development of $\mathrm{HCC}^{15}$. Some studies have found that METTL3 can promote the translation of the oncogenes EGFR and TAZ to promote the growth and invasion of human lung cancer cells ${ }^{16}$. However, research on the functions of m6A in PC is still limited. It has been found that WTAP is highly expressed in PC and associated with clinicopathological features, but related mechanisms have not been reported ${ }^{17}$. IGF2BP2 was found to regulate DANCR in PC through m6A modification to promote stemness-like properties and pathogenesis ${ }^{18}$. The exploration of m6A RNA methylation regulators in PC is still in its infancy. It is urgent to identify how the proteins regulating RNA methylation in PC are themselves regulated to further clarify their function and prognostic value. We classified PC patients according to the overall expression pattern of m6A RNA methylation regulators in the Cancer Genome Atlas (TCGA) database and constructed a risk score based on the least absolute shrinkage and selection operator method. We identified a crucial target, IGF2BP2, that mediates the malignant progression of PC and promotes B3GNT6 mRNA stability, contributing to further progression of PC.

\section{Methods}

\section{Clinical PC specimens}


PC together with counterpart adjacent normal tissue samples were obtained from department of General Surgery, the First Affiliated Hospital of Soochow University. The research was ratified by the Ethical Committee of the first affiliated hospital of Soochow University and written informed consent were successively obtained from all participants before the study.

\section{Cell culture}

Human CFPAC1 and PANC1 PC cells were purchased from GenePharma Technology Co., Ltd. and used in our research. These cells were cultured in Dulbecco's modified Eagle's medium supplemented with $10 \%$ foetal bovine serum (FBS) in an incubator with a $5 \% \mathrm{CO}_{2}$ atmosphere at $37^{\circ} \mathrm{C}$. All cells were free of bacterial and mycoplasma contamination.

\section{Transfection}

The target mRNA interference constructs and the NC construct si-NC were co-transfected with Lipofectamine 2000 reagent (Invitrogen, USA) to knock down mRNA and protein expression in PC cells; their sequences are shown in Supplementary table.

\section{RNA extraction and quantitative real-time polymerase chain reaction (qRT-PCR)}

Total RNA was extracted with TRIzol from PC cells and tissues. All primers used for qRT-PCR were designed and blasted in the National Center for Biotechnology Information database. RNA was reverse transcribed to CDNA with Vazyme reverse transcriptase following the protocol, and PCR was performed using SYBR Green qPCR Master Mix (GenePharma, China) in an ABI CFX Connect real-time PCR system (Bio-Rad, Hercules, USA) in triplicate. Human $\beta$-actin was used as the internal control for mRNA expression. The primer sequences are shown in Supplementary table.

\section{CCK-8 proliferation assay}

CCK-8 assay was used to detect the proliferation ability of PC cells. Transfected cells were seeded in 96well plates at a concentration of $5^{\star} 10^{3}$ cells per well. After cultured for $0,24,48,72$ and 96 hours under the same conditions, PC cells were incubated with diluted CCK-8 reagent (Dojindo Laboratories, Japan) following the instructions. Then, the absorbance was measured in a microplate reader at a wavelength of $450 \mathrm{~nm}$.

\section{EdU assay}

An EdU kit (RiboBio, China) was used to determine the proliferation capability. In brief, after stable transfection for 48 hours, cells were incubated with $5 \mu \mathrm{M}$ EdU reagent diluted with DMEM for 3 hours. Then, cells were permeabilized with $0.5 \%$ Triton X-100 for 20 minutes prior to fixation with $4 \%$ paraformaldehyde for 30 minutes. Apollo and DAPI dyes were used to stain DNA and then nuclei. Images of EdU- and DAPI-positive cells were acquired under a fluorescence microscope.

\section{Colony formation assay}


To verify the effect of PC cell colony formation ability, transfected cells were seeded into 6-well plates at a density of 5000 cells per well and distributed evenly by pipetting. After 14 days, the cells were washed with PBS twice, fixed with $4 \%$ paraformaldehyde for 30 minutes and stained with $1 \%$ crystal violet for 30 minutes. Eventually, the cells were imaged, and the colonies were counted.

\section{Transwell invasion assay}

To evaluate the cell migration ability, cells were cultured in serum-free medium in the upper chamber of a Transwell plate (Corning, USA), and $700 \mu$ l of DMEM containing $10 \%$ FBS was added to the lower chamber. After 48 hours, the cells were fixed and stained with crystal violet for 20 minutes, and the cells that migrated through the upper chamber membrane were counted. Images were acquired by microscopy.

\section{Luciferase reporter assay}

The B3GNT6 fragment containing the possible IGF2BP2 protein binding site and the corresponding mutated fragment were synthesized and cloned into the pmirGLO vector (Promega, Madison, USA) to synthesize the vectors named B3GNT6-WT and B3GNT6-Mut. The synthesized vectors were separately co-transfected with IGF2BP2-si or si-NC into 293T cells. After co-incubation for 24 hours, cells were collected, and luciferase activities were measured with a Dual Luciferase Reporter Assay kit (Promega, China).

\section{m6A IP and IGF2BP2 IP assays}

Immunoprecipitation assays of m6A and IG2BP2 were performed with an RIP kit (BersinBio Biotech, China). In brief, approximately $1 * 10^{7}$ treated cells were collected, and the cells were lysed for 30 minutes. DNA impurities were removed with DNase, the supernatant was collected after centrifugation, and the supernatant was then incubated with anti-m6A and anti-IGF2BP2 primary antibodies with overnight at $4^{\circ} \mathrm{C}$ in a vertical orientation. Then, magnetic beads were added for incubation for 1 hour. After washed, RNA was extracted, and target gene expression was detected by qRT-PCR.

\section{Quantification of overall m6A RNA methylation}

We used an EpiQuik m6A RNA Methylation Quantification Kit (colorimetric) (Epigentek, USA) to quantify the overall m6A level. First, RNA was extracted following the instructions, and the RNA concentration was adjusted for an initial input amount of approximately $200 \mathrm{ng}$. Then, the RNA and standard NC were bound to the positive control wells to assay and capture RNA. After washing, the detection antibody and enhancer solution were added; the wells were then washed twice, and colour developing solution was added prior to measurement of the absorbance. The values were calculated using linear regression equations and are presented as a standard region graph.

\section{3-DAA demethylation treatment}

The overall methylation inhibitor 3-DAA (Cayman, USA) was used to inhibit RNA methylation levels. After inoculation, cells were treated with $10 \mathrm{ug} / \mathrm{mL} 3-\mathrm{DAA}$ for 48 hours, then cells were collected for mRNA and protein analysis. 


\section{IHC analysis}

Paraffin sections of pancreatic tissue were prepared and baked in an oven at $65^{\circ} \mathrm{C}$ for 30 minutes. After routine dewaxing, hydration and antigen repair, sections were sealed with $5 \%$ goat serum for 30 minutes, placed in a wet box and incubated overnight with the primary antibody at $4^{\circ} \mathrm{C}$. The next day, sections were incubated with the secondary antibody for 30 minutes, incubated with DAB for colour development for 3 minutes, stained with haematoxylin for 3 minutes and washed for 30 minutes. Sections were sealed with neutral gum, and images were acquired with a microscope.

\section{IF detection assay}

After dewaxing, hydration and antigen repair, paraffin sections were blocked with immunostaining blocking solution for 1 hour, incubated with the primary antibody at $4^{\circ} \mathrm{C}$ overnight, and then incubated with the secondary antibody for 1 hour. After restaining with DAPI, sections were sealed with an antifluorescence quenching agent and imaged under a fluorescence microscope.

Treated PC cells were prepared as cell slides and were then fixed, permeabilized, blocked with blocking solution for 1 hour, incubated with the primary antibody at $4^{\circ} \mathrm{C}$ overnight, and then incubated with the secondary antibody. After nuclear staining with DAPI, sections were sealed and imaged.

\section{Western blot analysis}

We used a RIPA mixture containing 1\% PMSF lysate to lyse and obtain total protein from transfected cells. A BCA protein detection kit (Thermo Scientific Pierce, USA) was used to determine the protein concentration, and proteins were then separated. The electrophoresis conditions were $80 \mathrm{~V}$ for 30 minutes and $120 \mathrm{~V}$ for 1 hour, followed by transfer to polyvinylidene difluoride membranes in transfer buffer at $200 \mathrm{~mA}$ for 1.5 hours. Then, membranes were blocked in $5 \%$ skim milk at $37^{\circ} \mathrm{C}$ for 1 hour. After washing with PBST, membranes were incubated first with primary antibodies overnight at $4^{\circ} \mathrm{C}$ and then with an HRP-labelled goat anti-rabbit secondary antibody for 1 hour at room temperature. After washing 3 more times, immunoreactions were visualized with an electroluminescence detection system, and the data were saved and analysed by ImageJ. ACTB was used as a housekeeping gene.

\section{Animal experiment}

To evaluate the effect of IGF2BP2 on the tumour formation ability in vivo, $2 * 10^{6}$ cells were injected into the axilla of nude mice for subcutaneous tumour formation. After 9 days, modified IGF2BP2-si or NC was injected into the tumours every 3 days for 3 weeks. Tumour size was measured every 3 days. Finally, the subcutaneous tumours were excised and weighed to compare the tumour sizes, and protein expression was detected by immunohistochemistry and immunofluorescence. The care of laboratory animals was in accordance with the guidelines and ethical requirements of the Laboratory Animal Centre of Soochow University.

\section{Downloading of the PC dataset in TCGA}


We used the R package TCGAbiolinks to download the PC transcriptome data from the TCGA database and combined these data with healthy human tissue transcriptome data in GTEx to explore differential gene expression in PC. Patients with rational survival data and clinicopathological characteristics were included in the analysis. For group analysis, the median value was used as the cut-off value.

\section{Clinical bioinformatic analysis}

We employed the R software package ConsensusClusterPlus to divide PC patients into two subtypes (PA1 and PA2) according to the expression of the regulators and used principal component analysis (PCA) to evaluate the difference in the gene expression distribution between the two clusters. Additionally, we used the $\mathrm{R}$ software package limma to conduct differential gene expression analysis on the subgroups, where fold change $(F C)>2$ and $p$ value $<0.05$ were used as the cut-off values for DEGs, KEGG and GO enrichment analyses were performed with the above DEGs. The risk score was calculated using the LASSO-Cox regression algorithm, and four regulators were identified according to the minimum criteria and the correlation with survival prognosis.

\section{Statistical Analysis}

We used GraphPad 8.0 for charting. For statistical analysis, two-tailed Student's t-test between two groups and one-way ANOVA analysis of variance test for multiple groups comparison were performed by SPSS Statistic 26 for windows. The Kaplan-Meier method was used for survival analysis. Data were reported as mean \pm SD from three duplicate experiments. Data was considered to be statistically significant when $p$-value $<0.05$.

\section{Results}

\section{Overall expression pattern of m6A regulators}

Considering that methylation regulators perform different biological roles, we first analysed the expression of 15 regulators in PC and found that compared with normal pancreatic expression profiles in the GTEx database, the regulators were generally upregulated in TCGA, a pattern that was also observed in the GEO database (GSE15471) (Fig. 1a-b). Spearman correlation analysis showed a close relationship between the factors (Fig. 1c). Analysis of the STRING database also revealed a positive correlation between multiple regulators (Fig. 1d). Genome CNV is a form of genomic variation that usually causes large DNA fragments to generate an abnormal number of copies via mechanisms mainly including deletions, insertions, replication errors, and compound multi-site mutations. Previously, according to DGV data, CNV was determined to be present in nearly 2/5 tumour-related genes. Our CNV analysis also showed a general positive correlation between CNV and RNA expression (Supplementary Figure), together with clinicopathological features such as tumour size (Fig. 1e), suggesting that CNV is an important element leading to the upregulation of regulators and the progression of PC. 


\section{Identification of two subgroups of PC by Consensus clustering}

To visualize the specific functions of m6A methylation regulators, we removed normal pancreatic samples and PC samples with no suitable clinical data, and used the ConsensusClusterPlus R package to perform consensus clustering. According to the expression similarity of the regulators, the cumulative distribution function (CDF) of our data seemed to be ideal when $\mathrm{K}=2$, and the PA1 and PA2 subgroups were then defined (Fig. 1f-g). We compared the clinicopathological characteristics of PA1 and PA2 and found them to be consistent with the above expectations. K-M survival analysis showed significant differences between the two subgroups (Fig. 1h).

The edgeR software package was used to analyse the difference between the expression profiles of PA1 and PA2, and the DEGs were selected based on the criteria $\| \operatorname{logFC} \geq 1$ and $p$ value $\leq 0.05$. Finally, 2681 genes were identified; 724 genes were upregulated in PA1, and 1957 genes were upregulated in PA2. KEGG and GO enrichment analyses showed that the DEGs were mainly enriched in metabolic or immune pathways (Fig. 2a-b), such as Cytokine - cytokine receptor interaction and MAPK signaling pathway, indicating that mRNA methylation affects multiple biological processes.

\section{Analysis of associations between m6A methylation regulators and clinicopathological characteristics}

To further clarify the role of the regulators in survival, we conducted univariate Cox regression analysis. Among the 15 regulators, 5 were related to prognosis: IGF2BP2 $(H R=2.19)$, IGF2BP3 (HR = 1.53), METTL3 $(\mathrm{HR}=0.53), \mathrm{ALKBH} 5(\mathrm{HR}=0.6)$ and KIAA1429 (HR = 1.55) (Fig. 2c). Survival models based on high reliability and LASSO regression are widely used to screen prognostic genes from high-dimensional data; thus, we established risk characteristics and performed LASSO-Cox regression analysis to calculate the risk score, and found that KIAA1429, METTL3, IGF2BP2 and ALKBH5 were the main contributors (Fig. 2de). According to the median risk score, low-risk patients had higher survival status (Fig. 2f). The respective survival rate analysis showed higher IGF2BP2 contribute to lower survival rate (Fig. $2 \mathrm{~g}$ ). Heatmap shows that ALKBH5 and METTL3 were upregulated in the low-risk group, while IGF2BP2 and KIAA1429 were upregulated in the high-risk group (Fig. $2 \mathrm{~h}$ ). We evaluated the correlations between the risk subgroups and the clinicopathological features. Univariate and multivariate Cox regression analyses showed that the risk score $(p<0.05)$ and lymph node metastasis $(p<0.05)$ were highly significant (Fig. 2ij). In summary, we believe that the accuracy of m6A methylation regulators for predicting the prognosis of PC was further corroborated. Considering that IGF2BP2 is highly expressed in PC, with a high HR, and is strongly independently associated with prognosis, we selected it as the main regulator for subsequent studies.

\section{IGF2BP2 is a credible molecular prognostic marker in PC}


TCGA analysis revealed high expression of IGF2BP2 in PC (Fig. 3a); our IHC analysis also verified its overexpression (Fig. 3b), and qRT-PCR revealed that high expression of IGF2BP2 was detected in CFPAC1 and PANC1 cells compared with normal pancreatic cells (Fig. 3c). We then sought to investigate some specific reasons for high IGF2BP2 expression. TCGA data analysis showed that increasing CNV contributed to a higher mRNA level (Fig. 3d), while the DNA methylation level was negatively correlated with the mRNA expression (Fig. 3e), indicating that DNA methylation and CNV were indispensable cooperative factors for high IGF2BP2 expression. These results indicated that IGF2BP2 was a marker of unfavourable prognosis in $\mathrm{PC}$ patients.

\section{IGF2BP2 promotes PC cell proliferation and migration}

For further investigation of the biological function of IGF2BP2 in PC, we knocked down the expression of IGF2BP2 in CFPAC1 and PANC1 cells with siRNA and named the resulting cell IGF2BP2-si-1 and IGF2BP2si-2; the knockdown efficiency was confirmed by qRT-PCR and western blot (Fig. 3f-h). CCK-8 and EdU assay incorporation assays indicated that silencing IGF2BP2 obviously suppressed the proliferation of PC cells (Fig. 4a-d). Transwell assay was applied to explore the cell migration ability; as shown, fewer cells in the siRNA group than in the control group passed through the membrane of the upper compartment (Fig. 4e). The colony formation assay also showed that inhibition of IGF2BP2 decreased cell viability (Fig. 4f). These results illustrate that IGF2BP2 exerts oncogenic effects in PC.

\section{IGF2BP2 regulates B3GNT6 expression}

As an RNA binding protein, IGF2BP2 has been proven to bind to a variety of RNAs to regulate their expression. To identify potential target genes of IGF2BP2, we analysed IGF2BP2 CLIP data and identified 6249 targets with relatively high associativity. Subsequently, PC patients were divided into a high expression group and a low expression group according to the expression level of IGF2BP2, and differential gene expression analysis was conducted between the groups, identifying 172 genes that may be regulated by IGF2BP2. In these patients, we identified 8335 DEGs between PC and normal tissue, among which nine were common highly reliable genes (Fig. 5a). qRT-PCR confirmed that after knockdown of IGF2BP2, some genes were downregulated to varying degrees; among these genes, B3GNT6, DHRS9 and ALPP had the largest decreases (Fig. 5b). The IGF2BP2 and m6A RIP results showed that B3GNT6 was more highly enriched than DHRS9 and ALPP (Fig. 5C). B3GNT6 is a beta-1,3-N-acetylglucosaminyl transferase that adds an $\mathrm{N}$-acetylglucosamine moiety to $\mathrm{N}$-acetylgalactosamine-modified serine or threonine residues to affect biosynthesis and metabolism; together, these results indicate that B3GNT6 may be the downstream target of IGF2BP2. TCGA data analysis indicated that B3GNT6 was characteristically upregulated in PC (Fig. 5d), and Spearman correlation analysis also showed the coexpression of IGF2BP2 and B3GNT6 (Fig. 5e).

\section{Inhibition of B3GNT6 decreased the proliferative ability of PC cells}


The interference construct B3GNT6-si was transfected into PANC1 and CFPAC1 cells to knock down B3GNT6 expression, CCK-8 and EdU incorporation assays were applied for cell proliferation analysis. The results indicated that silencing B3GNT6 overtly inhibited the proliferation of PC cells (Fig. $5 f-i)$. In addition, the Transwell assay showed that fewer cells in the B3GNT6-si group than in the NC group passed through the Transwell compartment membrane (Fig. 5j); thus, we believe that B3GNT6 can regulate the proliferation and migration capacities of PC cells. Additionally, the colony formation assay showed that knockdown of B3GNT6 decreased cell viability (Fig. 5k). These results indicate that B3GNT6 can exert oncogenic effects in PC.

\section{IGF2BP2 recognizes m6A methylation on B3GNT6 and increases its stability}

To further identify the underlying mechanism of IGF2BP2-mediated B3GNT6 expression, we sought to determine whether and how IGF2BP2 interacts with B3GNT6 to affect its expression. IGF2BP2, a member of the IGF2BP family, has been recently proven to be involved in the RNA methylation pathway and to function as an methylation reader to enhance RNA stability. To determine whether B3GNT6 is subject to RNA methylation (m6A) such that methylated B3GNT6 mRNA is recognized by IGF2BP2, we first detected the overall m6A content and found that IGF2BP2 knockdown contributed to a lower m6A level (Fig. 6a); then, a RIP assay with IGF2BP2 and an anti-m6A antibody identified 4-fold enrichment of B3GNT6 compared to the IgG control (Fig. 5c). IGF2BP2 knockdown reduced the m6A level on B3GNT6 compared to that in control cells (Fig. 6b). RNA m6A methylation occurs primarily at RRACH sequences, and we hypothesized that the RRACH sequence in B3GNT6 mRNA was also modified by m6A methylation. A dual luciferase reporter assay was conducted, and the results revealed that among the feasible binding sites, GGACA (nt 1219-1223) is the only site for m6A modification (Fig. 6c), while other predicted sites did not exhibit significant binding (Table 1).

Considering that m6A methylation can impact RNA stability, we sought to evaluate B3GNT6 mRNA stability. PANC1 cells were transfected with IGF2BP2-siRNA or the NC, treated with 3-DAA for 48 hours, and then treated with actinomycin $D$ at $2 \mu \mathrm{g} / \mathrm{ml}$ before total RNA was extracted. As shown, B3GNT6 mRNA decay in the IGF2BP2-siRNA-treated cells was faster than that in the NC-treated cells (Fig. 6d-e), suggesting that IGF2BP2 can increase B3GNT6 mRNA stability and contribute to the high B3GNT6 protein level. Together, these results suggest that similar to other mRNAs, B3GNT6 can undergo m6A methylation, and IGF2BP2 functions as a reader of methylated B3GNT6 to increase its stability. IF staining revealed that IGF2BP2 and B3GNT6 had similar expression trends in PC tissue (Fig. 6f), and knockdown of IGF2BP2 led to an obvious decrease in the B3GNT6 protein level (Fig. 6g).

\section{IGF2BP2 promotes tumorigenesis in vivo}

Subcutaneous tumorigenesis experiments in mice showed that knockdown of IGF2BP2 contributed to a decreased tumour size (Fig. 6h-i) and tumour weight (Fig. 6j), showing the significant ability of IGF2BP2 to promote tumour formation. We then evaluated the B3GNT6 expression level by IHC and IF, finding that knockdown of IGF2BP2 contributed to a lower B3GNT6 expression level (Fig. 6k-I). 
Together, these data indicate that B3GNT6 is a downstream target of IGF2BP2 and serves as an oncogene in PC.

\section{Discussion}

We conducted a comprehensive expression profiling analysis of 15 methylation regulators and found overall high expression in PC samples; in addition, we found strong correlations between regulator proteins. Because a writer promotes mRNA methylation while an eraser mediates demethylation. Retrospective study found that gene CNV was positively correlated with gene expression; the higher the copy number was, the shorter the survival time of patients, which is consistent with the high regulator expression in PC. We speculate that an increased copy number leads to increased gene stability and expression. In addition, we analysed the DNA methylation level and reached the opposite conclusion. We believe that a high gene copy number and low DNA methylation level synergistically promote m6A methylation in PC, although this hypothesis still needs further experimental verification. We divided PC samples into two subgroups and performed differential gene expression analysis. Enrichment analysis showed that the gene sets were mainly enriched in the immune pathway. Then, we used LASSO-Cox regression analysis to construct a methylation model and obtained a risk score, finding that the survival rate in the high-risk group was significantly lower than that in the low-risk group. Finally, we selected the potential m6A downstream target gene B3GNT6 based on the RIP and dual luciferase reporter assays. Subsequent in vivo and in vitro experiments were performed to verify the gene methylation modification.

The mortality of PC varies greatly in different regions. In addition, due to late detection and the scarcity of effective treatments, the survival rate is generally low ${ }^{19}$. Currently, effective treatment for PC is still based on traditional surgical treatment, but $80 \%-90 \%$ of patients have residual tumours and a poor survival rate $^{20}$. The occurrence and development of PC is very complex; it is a multi-factor, multi-step process involving environmental factors, dietary factors, lifestyle habits, genetic changes, gene expression patterns, molecular interactions, changes in signal transduction pathways, etc.; in addition, drug resistance and post-operative burden are considerable problems in clinical practice ${ }^{21}$. In regard to posttranscriptional modification, methylation is the most common epigenetic modification of RNA, and m6A is the most universal methylation modification ${ }^{9,22}$. To date, its various functions in cancer have gradually been revealed, although this knowledge is just the tip of the iceberg. By modifying the methylation of specific sites in mRNA, one m6A methylation regulator may have different functions in different cancers. m6A methylation is mainly enriched near stop codons in 3'-untranslated regions containing the consensus sequence RRACH $(R=A \text { or } G, A \text { is m6A modified, and } H=A, C \text { or } U)^{23}$;

m6A modifies and regulates different aspects of mRNA, including its structure, stability, splicing, nuclear export, translation, decay, etc., and is also involved in cell fate determination, cell cycle regulation, and cell differentiation. Among the regulators, m6A writers mainly increase the level of methylation on RNA, which is the key step in m6A modification. A variety of writer proteins have been confirmed, for example, WTAP, METTL3 and METTL14, which form a classic methylation complex to promote RNA methylation and 
localize to nuclear speckles ${ }^{24}$. As a reversible modification, m6A eraser has the opposite function of a writer to reduce the level of m6A modification, but may eventually contribute to similar functional results, because there are also m6A readers, a group of proteins that can recognize $\mathrm{m6A}$; these readers include YTH domain family homologous proteins YTHDF1, YTHDF2, YTHDF3 and YTHDC2 in the cytoplasm and YTHDC1 in the nucleus. YTHDF1 promotes the translation of m6A-methylated mRNA, while YTHDF2 accelerates the degradation of m6A-methylated mRNA ${ }^{25,26}$; thus, when writers or erasers cooperate with different readers, they can contribute to various biological functions, which also leads to the complexity of m6A modification ${ }^{27}$.

To date, m6A methylation has been found to function as a carcinogenic or tumour-suppressive mechanism in glioblastoma ${ }^{28}$, hepatocellular carcinoma ${ }^{15,29}$, and breast cancer ${ }^{30}$, but the function of m6A methylation in PC is still unknown. Previous studies have shown that YTHDF2 inhibits adhesion, invasion, migration and EMT through YAP signalling ${ }^{31}$. WTAP stabilizes Fak mRNA in PC to promote metastasis and gemcitabine resistance ${ }^{32}$, PIK3CB m6A methylation promotes the progression of PTENdeficient $P C$ by regulating the AKT signalling pathway ${ }^{33}$, but the pattern of methylation and the progression of malignancy in PC remain to be explored. Our results show that m6A methylation contributes to the classification of PC and is closely related to the clinicopathological characteristics of PC. METTL3 was originally identified as a methyltransferase responsible for m6A modification ${ }^{34}$, and increasing evidence shows that it functions in MRNA shearing ${ }^{35}, 3^{\prime}$-UTR modification ${ }^{36}$, translational regulation ${ }^{26}$ and decay ${ }^{23}$. YTHDF1 has a conserved m6A binding domain and preferentially binds m6Amodified RNA sites. Studies have found that YTHDF1 interacts with initiation factors to enhance mRNA translation and protein synthesis or inhibits $T$ cell function to promote escape from immune surveillance ${ }^{37}$. In contrast to YTHDF2's ability to promote mRNA attenuation, IGF2BP2 can promote the stability and maintenance of its target mRNA in an m6A-dependent manner ${ }^{38,39}$, which is usually achieved after binding to an mRNA stabilizer.

In this study, we found that as a stabilizer of m6A methylation, IGF2BP2 promotes B3GNT6 mRNA stability and protein expression. In theory, the reader, eraser and writer should have different expression patterns due to their different functions. However, our research found that the expression patterns of most regulators, whether they promote methylation or demethylation, tended to be the same. We speculated that on the one hand, there may be a proportional relationship. For example, a ratio of the methylation/demethylation regulators exceeding a certain range will promote cancer or suppress cancer. On the other hand, different regulators have different affinities for the same gene and contributions to abnormal downstream activities caused by m6A methylation ${ }^{39,40}$. Third, there may be other molecules affecting m6A methylation modification; indeed, these still need to be further verified.

\section{Conclusions}

In summary, we have confirmed the elevated m6A level and revealed the significant effect of RNA methylation in PC. Besides we authenticate a novel gene IGF2BP2 is a credible marker and upregulated in 
PC, m6A-IP and dual luciferase reporter indicate that it promotes the m6A methylation level of B3GNT6 mRNA to contribute to the deterioration of PC, indicating that IGF2BP2 is a potential prognostic marker and therapeutic target in PC progression. Hence, our study provides some evidence for research of RNA methylation in PC.

\section{Abbreviations}

3-DAA 3-deazaadenosine

CNV copy number variation

CCK-8 Cell Counting Kit-8

DEGs differentially expressed genes

EdU 5-Ethynyl-2-deoxyuridine

GTEx Genotype-Tissue Expression

IHC Immunohistochemical

IF Immunofluorescence

m6A N6-methyladenosine

NC negative control

RIP RNA immunoprecipitation

\section{Declarations}

\section{Acknowledgements}

The authors thank Genepharma (Suzhou, China) for technical support and equipment employment. Thank Zhi Cao, Yubin Zhao, Rongkuan Hu, Xiangyong Chen for theoretical guidance. Also we thank Hong Zhou, Pengcheng Cao for experimental skills. We thank the Animal Experiment Center of Soochow University for for taking care of the animals.

\section{Availability of data and materials}

The data that support this study are available upon request. Bioinformatics data was downloaded from TCGA and GEO database, Supplementary Figures and Tables are provided as a source data file. 


\section{Declarations}

\section{Ethics approval and consent to participate}

All animal experiments were performed according to the guide for the Laboratory Animal Centre of Soochow University. The care of laboratory animals was in accordance with the guidelines and ethical requirements of the Laboratory Animal Centre of Soochow University.

\section{Availability of supporting data}

Not applicable.

\section{Competing interests}

The authors declare no competing financial interests.

\section{Funding}

This research was supported by the Youth Science Foundation of National Natural Science Foundation of China (No. 81802365)

\section{Authors' contributions}

Xiaofeng Xue and Lei Qin contributed to the conception and design of the article.

Pei Cao, Ding Sun and Weigang Zhang contributed to the acquisition of the data.

Pei Cao and Yufan Wu contributed to the implementation of the experiment.

Zuxiong Tang, Junyi Qiu and Ding Sun contributed to the acquisition of specimens.

Pei Cao, Yufan Wu and Xiaofeng Xue contributed to the bioinformatics analysis.

Xiaofeng Xue, Pei Cao and Junyi Qiu contributed to the analysis and interpretation of the data.

Xiaofeng Xue, Lei Qin and Pei Cao contributed to the writing, review, and revision of the paper.

\section{Consent for publication}

All authors have read and approved the final manuscript.

\section{Publisher's Note}

Springer Nature remains neutral with regard to jurisdictional claims in published maps and institutional affiliations.

\section{Contributor Information}


Pei Cao, Email: 2738391149@qq.com

Yufan Wu, Email: kunshanwuyufan@163.com

Ding Sun, Email: sunding0126@163.com

Weigang Zhang, Email: 598745753@qq.com

Junyi Qiu, Email: qiujunyi1990@163.com

Zuxiong Tang, Email: tangzuxiong@suda.edu.cn

Xiaofeng Xue, Phone: +86-15606132969, Email: xfxue@suda.edu.cn

Lei Qin, +86-0512-67780178, Email: qinlei@suda.edu.cn

\section{References}

1. Vincent A, Herman J, Schulick R, Hruban RH, Goggins M. Pancreatic cancer. Lancet. 2011;378:60720. doi:10.1016/S0140-6736(10)62307-0.

2. Chen W, et al. Cancer statistics in China, 2015. CA Cancer J Clin. 2016;66:115-32. doi:10.3322/caac.21338.

3. Zhu H, Li T, Du Y, Li M. Pancreatic cancer: challenges and opportunities. BMC Med. 2018;16:214. doi:10.1186/s12916-018-1215-3.

4. Ligorio M, et al. Stromal Microenvironment Shapes the Intratumoral Architecture of Pancreatic Cancer. Cell 178, doi:10.1016/j.cell.2019.05.012 (2019).

5. Maitra A, Hruban RH. Pancreatic cancer. Annu Rev Pathol. 2008;3:157-88.

6. Feig C, et al. The pancreas cancer microenvironment. Clin Cancer Res. 2012;18:4266-76. doi:10.1158/1078-0432.CCR-11-3114.

7. Liu J, Harada BT, He C. Regulation of Gene Expression by N-methyladenosine in Cancer. Trends Cell Biol. 2019;29:487-99. doi:10.1016/j.tcb.2019.02.008.

8. Jia G, et al. N6-methyladenosine in nuclear RNA is a major substrate of the obesity-associated FTO. Nat Chem Biol. 2011;7:885-7. doi:10.1038/nchembio.687.

9. Zhao BS, Roundtree IA, He C. Post-transcriptional gene regulation by mRNA modifications. Nat Rev Mol Cell Biol. 2017;18:31-42. doi:10.1038/nrm.2016.132.

10. Zhao X, et al. FTO-dependent demethylation of N6-methyladenosine regulates mRNA splicing and is required for adipogenesis. Cell Res. 2014;24:1403-19. doi:10.1038/cr.2014.151.

11. Su R, et al. R-2HG Exhibits Anti-tumor Activity by Targeting FTO/mA/MYC/CEBPA Signaling. Cell 172, doi:10.1016/j.cell.2017.11.031 (2018).

12. Bartosovic M, et al. N6-methyladenosine demethylase FTO targets pre-mRNAs and regulates alternative splicing and 3'-end processing. Nucleic Acids Res. 2017;45:11356-70. 
doi:10.1093/nar/gkx778.

13. Cui Q, et al. mA RNA Methylation Regulates the Self-Renewal and Tumorigenesis of Glioblastoma Stem Cells. Cell Rep. 2017;18:2622-34. doi:10.1016/j.celrep.2017.02.059.

14. Barbieri I, et al. Promoter-bound METTL3 maintains myeloid leukaemia by mA-dependent translation control. Nature. 2017;552:126-31. doi:10.1038/nature24678.

15. Chen M, et al. RNA N6-methyladenosine methyltransferase-like 3 promotes liver cancer progression through YTHDF2-dependent posttranscriptional silencing of SOCS2. Hepatology. 2018;67:2254-70. doi:10.1002/hep.29683.

16. Lin S, Choe J, Du P, Triboulet R, Gregory RI. The m(6)A Methyltransferase METTL3 Promotes Translation in Human Cancer Cells. Mol Cell. 2016;62:335-45. doi:10.1016/j.molcel.2016.03.021.

17. Li B-Q, et al. WT1-associated protein is a novel prognostic factor in pancreatic ductal adenocarcinoma. Oncol Lett. 2017;13:2531-8. doi:10.3892/ol.2017.5784.

18. Hu X, et al. IGF2BP2 regulates DANCR by serving as an N6-methyladenosine reader. Cell Death Differ. 2020;27:1782-94. doi:10.1038/s41418-019-0461-z.

19. Karim-Kos HE, et al. Recent trends of cancer in Europe: a combined approach of incidence, survival and mortality for 17 cancer sites since the 1990s. Eur J Cancer. 2008;44:1345-89. doi:10.1016/j.ejca.2007.12.015.

20. Oberstein PE, Olive KP. Pancreatic cancer: why is it so hard to treat? Therap Adv Gastroenterol. 2013;6:321-37. doi:10.1177/1756283X13478680.

21. Kleeff J, et al. Pancreatic cancer. Nat Rev Dis Primers. 2016;2:16022. doi:10.1038/nrdp.2016.22.

22. Wang T, Kong S, Tao M, Ju S. The potential role of RNA N6-methyladenosine in Cancer progression. Mol Cancer. 2020;19:88. doi:10.1186/s12943-020-01204-7.

23. Meyer KD, et al. Comprehensive analysis of mRNA methylation reveals enrichment in $3^{\prime}$ UTRs and near stop codons. Cell. 2012;149:1635-46. doi:10.1016/j.cell.2012.05.003.

24. Liu J, et al. A METTL3-METTL14 complex mediates mammalian nuclear RNA N6-adenosine methylation. Nat Chem Biol. 2014;10:93-5. doi:10.1038/nchembio.1432.

25. Wang $X$, et al. N6-methyladenosine-dependent regulation of messenger RNA stability. Nature. 2014;505:117-20. doi:10.1038/nature12730.

26. Wang X, et al. N(6)-methyladenosine Modulates Messenger RNA Translation Efficiency. Cell. 2015;161:1388-99. doi:10.1016/j.cell.2015.05.014.

27. He L, et al. Functions of N6-methyladenosine and its role in cancer. Mol Cancer. 2019;18:176. doi:10.1186/s12943-019-1109-9.

28. Visvanathan A, et al. Essential role of METTL3-mediated mA modification in glioma stem-like cells maintenance and radioresistance. Oncogene. 2018;37:522-33. doi:10.1038/onc.2017.351.

29. Chen $M$, Wong C-M. The emerging roles of N6-methyladenosine (m6A) deregulation in liver carcinogenesis. Mol Cancer. 2020;19:44. doi:10.1186/s12943-020-01172-y. 
30. Cai X, et al. HBXIP-elevated methyltransferase METTL3 promotes the progression of breast cancer via inhibiting tumor suppressor let-7g. Cancer Lett. 2018;415:11-9. doi:10.1016/j.canlet.2017.11.018.

31. Chen J, et al. YTH domain family 2 orchestrates epithelial-mesenchymal transition/proliferation dichotomy in pancreatic cancer cells. Cell Cycle. 2017;16:2259-71. doi:10.1080/15384101.2017.1380125.

32. Li B-Q, et al. WT1 associated protein promotes metastasis and chemo-resistance to gemcitabine by stabilizing Fak mRNA in pancreatic cancer. Cancer Lett. 2019;451:48-57. doi:10.1016/j.canlet.2019.02.043.

33. Tian J, et al. N-methyladenosine mRNA methylation of regulates AKT signalling to promote PTENdeficient pancreatic cancer progression. Gut. 2020;69:2180-92. doi:10.1136/gutjnl-2019-320179.

34. Bokar JA, Shambaugh ME, Polayes D, Matera AG, Rottman FM. Purification and cDNA cloning of the AdoMet-binding subunit of the human mRNA (N6-adenosine)-methyltransferase. RNA. 1997;3:123347.

35. Liu N, et al. N(6)-methyladenosine-dependent RNA structural switches regulate RNA-protein interactions. Nature. 2015;518:560-4. doi:10.1038/nature14234.

36. Ke $\mathrm{S}$, et al. A majority of m6A residues are in the last exons, allowing the potential for $3^{\prime}$ UTR regulation. Genes Dev. 2015;29:2037-53. doi:10.1101/gad.269415.115.

37. Han D, et al. Author Correction: Anti-tumour immunity controlled through mRNA mA methylation and YTHDF1 in dendritic cells. Nature. 2019;568:E3. doi:10.1038/s41586-019-1046-1.

38. Huang $\mathrm{H}$, et al. Recognition of RNA N-methyladenosine by IGF2BP proteins enhances mRNA stability and translation. Nat Cell Biol. 2018;20:285-95. doi:10.1038/s41556-018-0045-z.

39. Li J, et al. N6-Methyladenosine Regulates the Expression and Secretion of TGF $\beta 1$ to Affect the Epithelial-Mesenchymal Transition of Cancer Cells. Cells 9, doi:10.3390/cells9020296 (2020).

40. Lin X, et al. RNA mA methylation regulates the epithelial mesenchymal transition of cancer cells and translation of Snail. Nat Commun 10, 2065, doi:10.1038/s41467-019-09865-9 (2019).

\section{Figures}


a

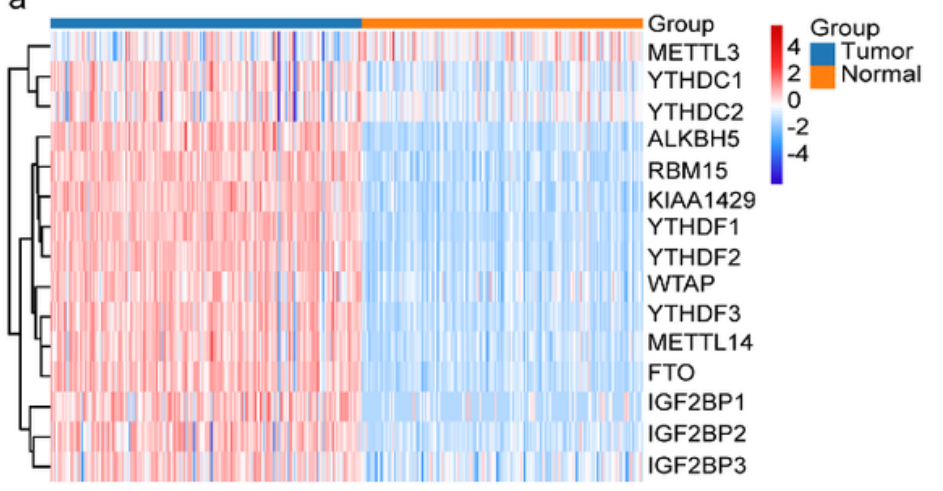

b

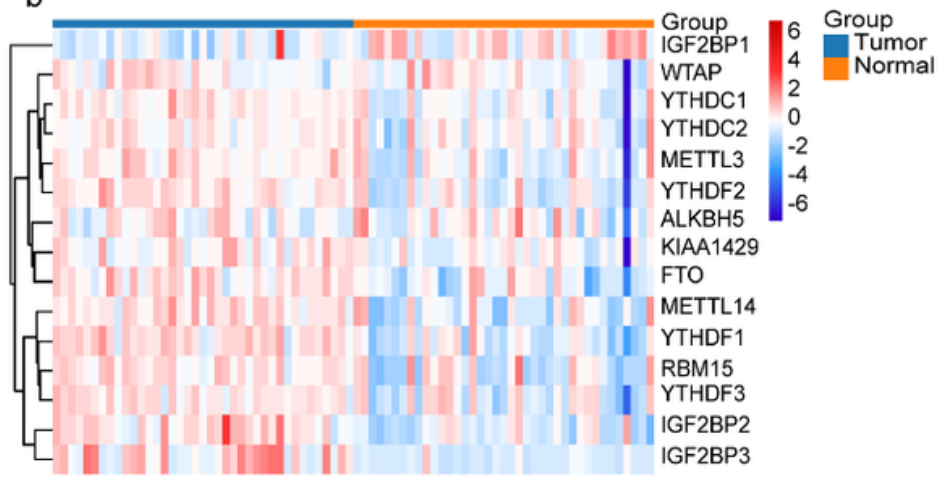

d
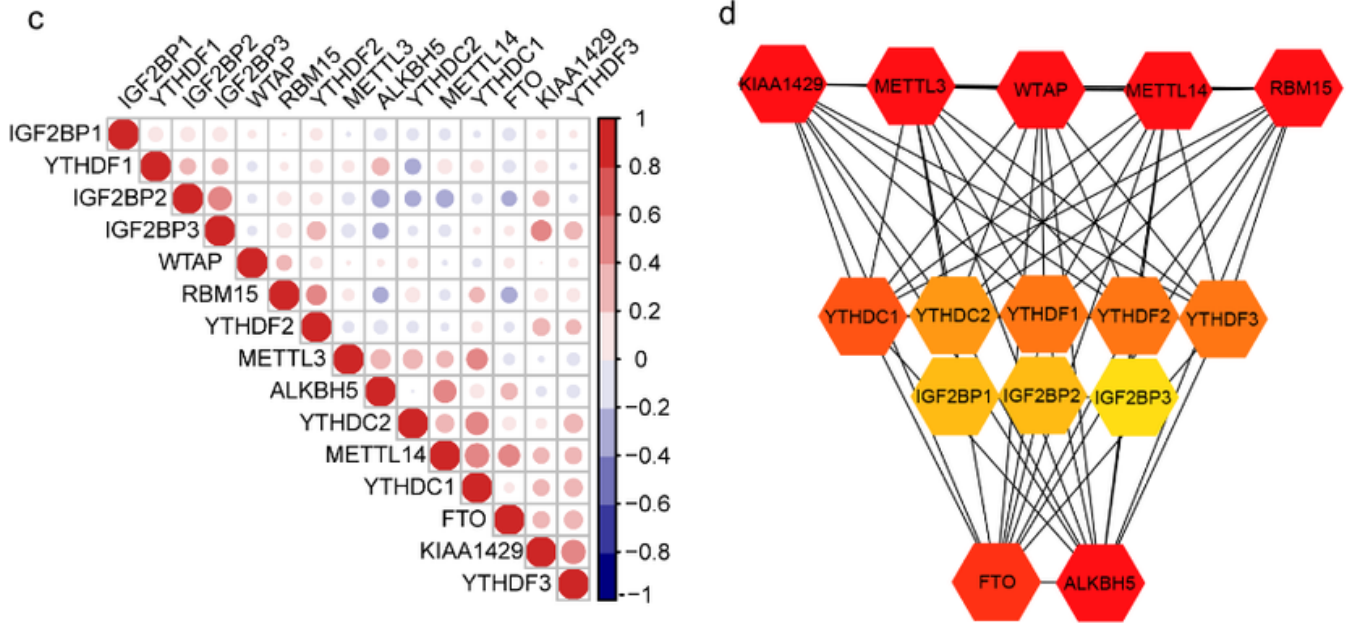

e

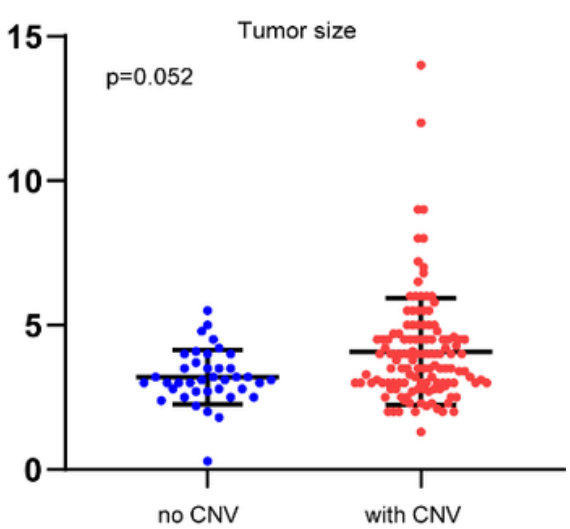

$\mathrm{h}$

consensus matrix $\mathrm{k}=2$

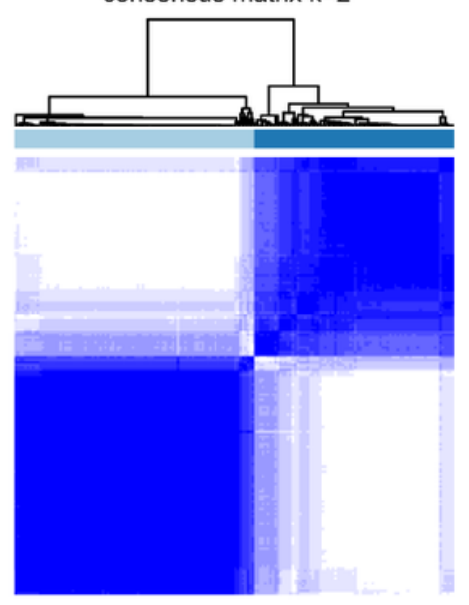

Survival proportions

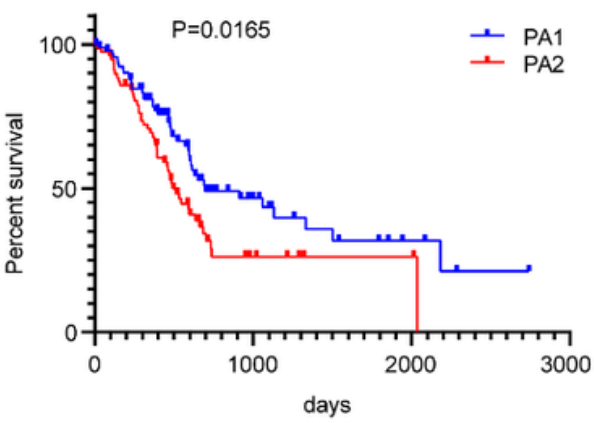

Figure 1

Expression of m6A methylation regulators and identification of consensus clusters by m6A regulators in PC. a-b The expression levels of 15 m6A RNA methylation regulators in PC in TCGA (a) and GEO (b) (red is up-regulated and blue is down-regulated). c Spearman correlation analysis of $15 \mathrm{~m} 6 \mathrm{~A}$ regulators in PC. $\mathrm{d}$ PPI network shows the interaction among 15 m6A regulators. e Relationship between overall CNV and tumor size. $\mathrm{f}$ Consensus clustering cumulative distribution function (CDF) for $\mathrm{k}=2$ to $10 \mathrm{~g}$ Consensus clustering matrix for $\mathrm{k}=2$. $\mathrm{h}$ Kaplan-Meier analysis for two subgroups. 

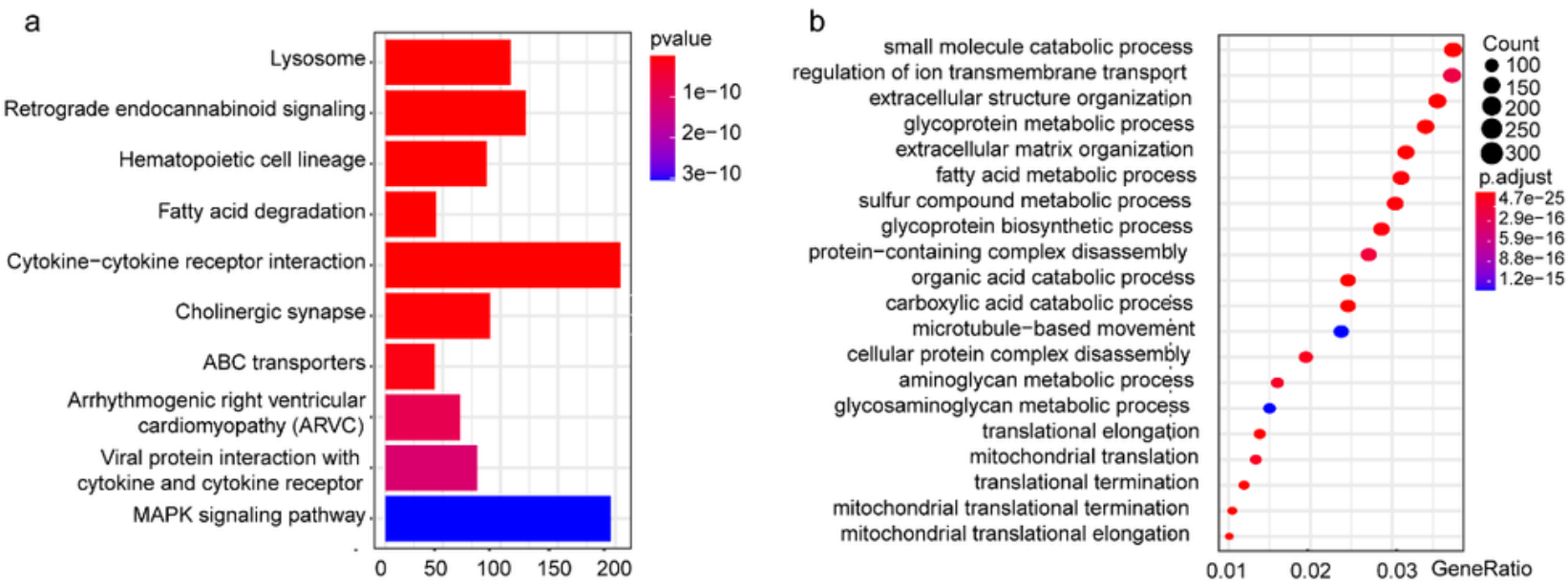

c

d
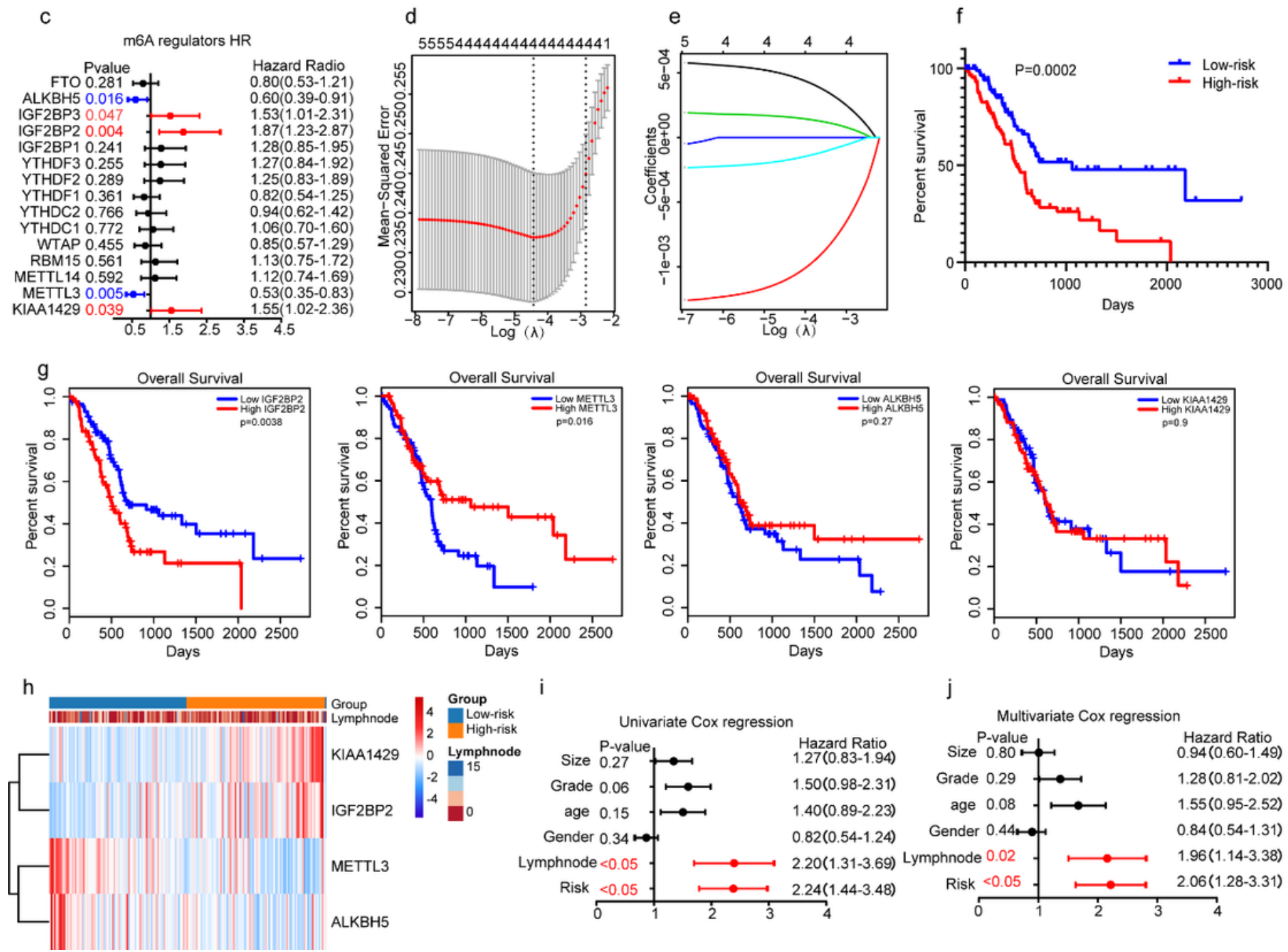

Figure 2

Enrichment analysis between subgroups and risk signature with four m6A RNA methylation regulators. ab KEGG and GEO analysis of the subgroups. c The process of building the signature containing $15 \mathrm{~m} 6 \mathrm{~A}$ RNA methylation regulators. d-e The coefficients calculated by multivariate Cox regression using LASSO are shown. $f$ Overall survival analysis between low- and high-risk groups stratified by the risk score. $\mathrm{g}$ Overall survival analysis of the four m6A regulators. $\mathrm{h}$ The heatmap shows the expression levels of the 
four m6A RNA methylation regulators and related clinicopathological features in low and high risk PC patients. i-j Univariate and multivariate Cox regression analyses of the association between some clinicopathological factors and overall survival in TCGA.

a

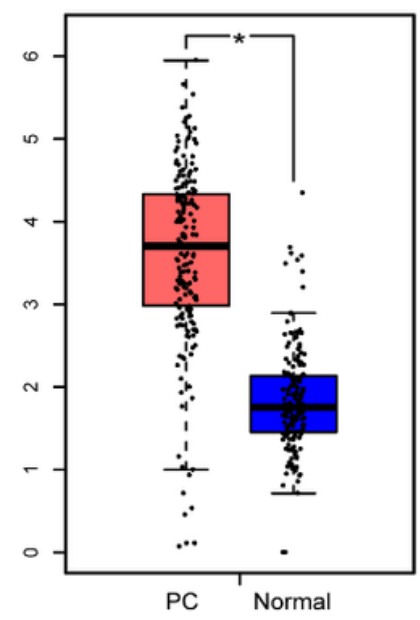

d

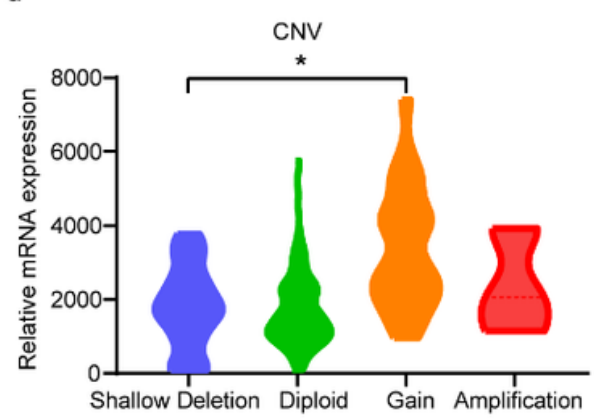

b

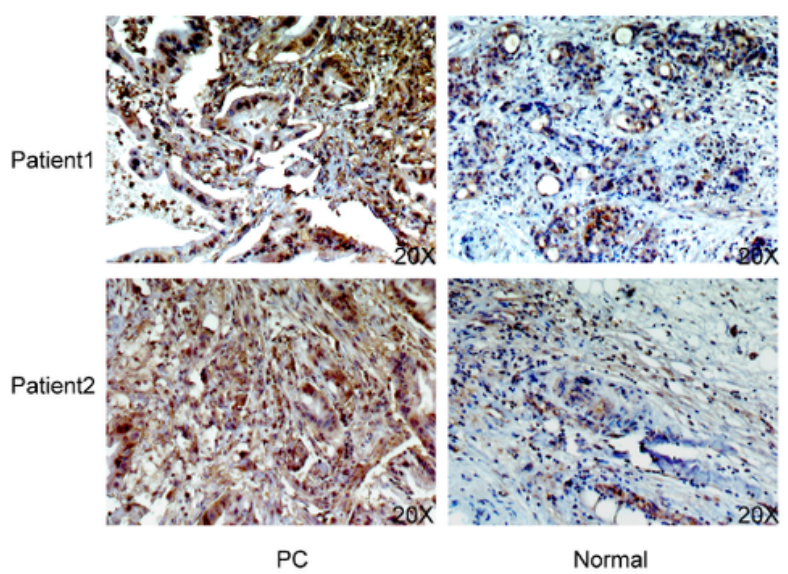

e

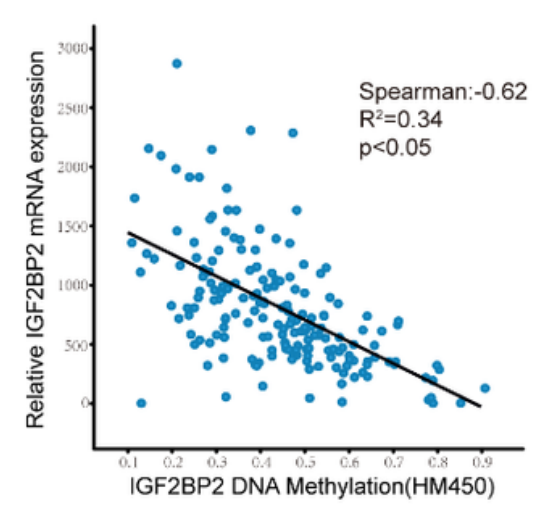

C

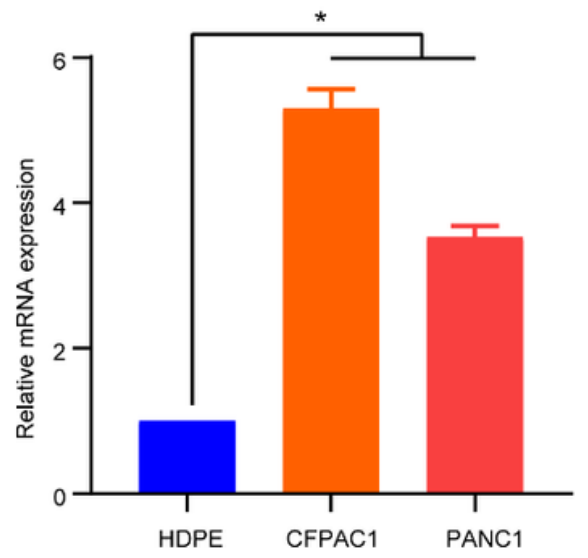

f

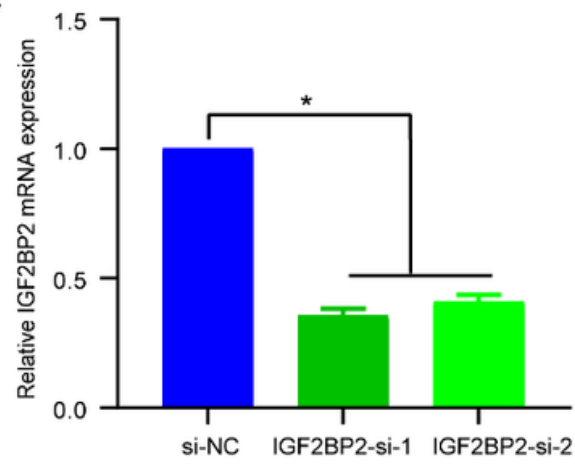

g

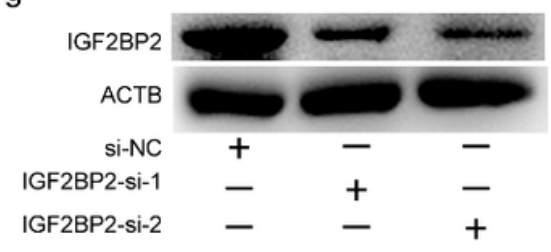

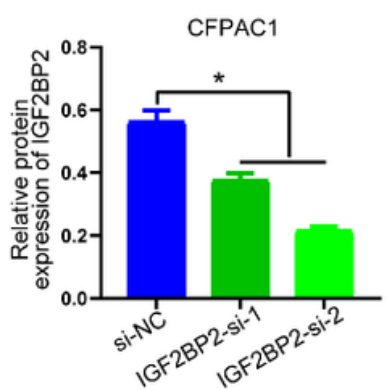
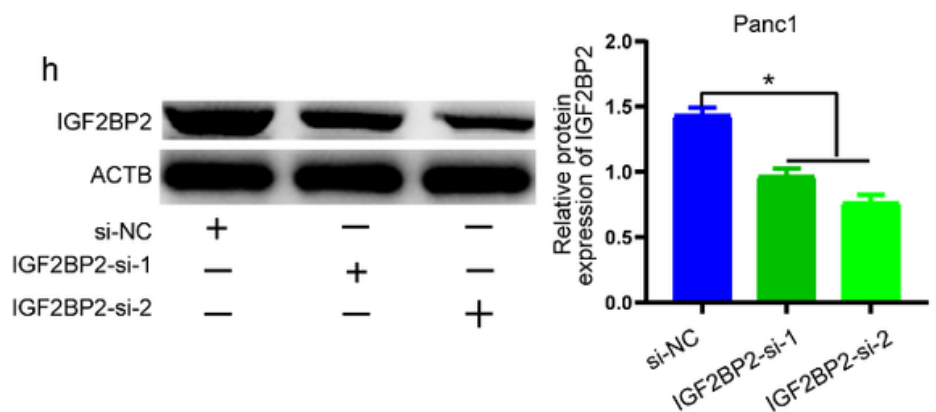

\section{Figure 3}

Expression of IGF2BP2 in pancreatic carcinoma. a Expression of IGF2BP2 mRNA in TCGA and GTEx database. b IHC (IGF2BP2)-stained paraffin-embedded sections verified the expression of IGF2BP2 protein in PC and normal tissue. c Expression of IGF2BP2 mRNA in different pancreatic cells. $d$ Relationship between different CNV types and IGF2BP2 expression level. e Relationship between IGF2BP2 DNA methylation and mRNA expression. $f$ IGF2BP2 mRNA expression in control and IGF2BP2-si groups. g-h IGF2BP2 protein expression in control and IGF2BP2-si groups. 

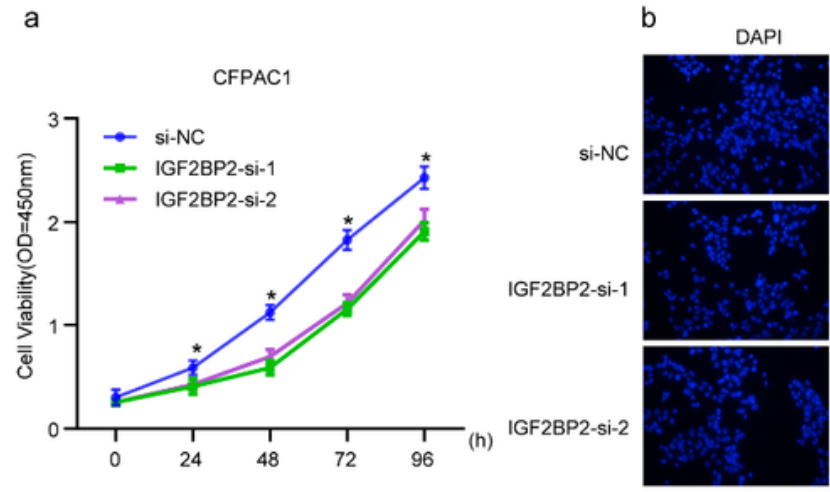

C

PANC1
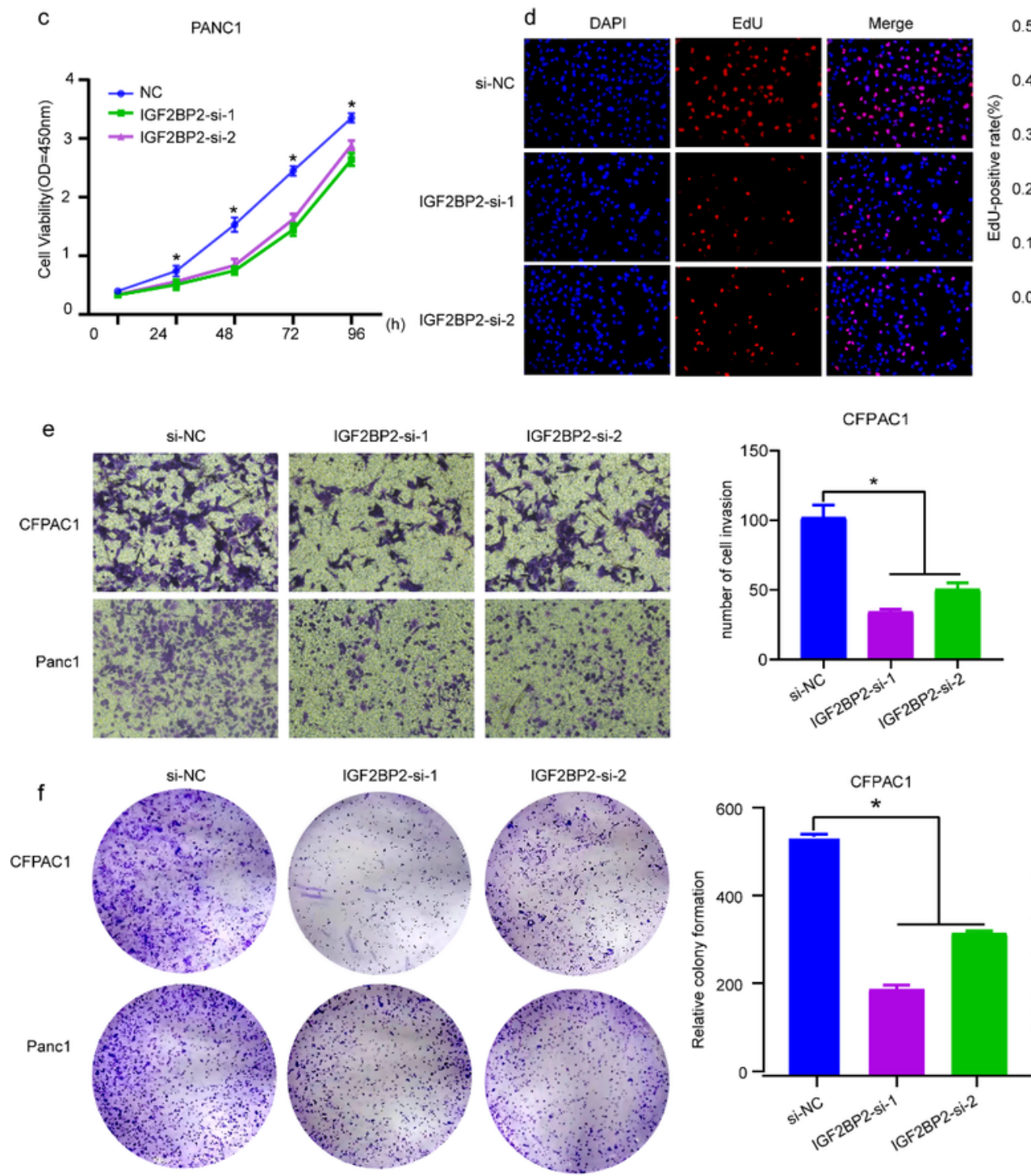
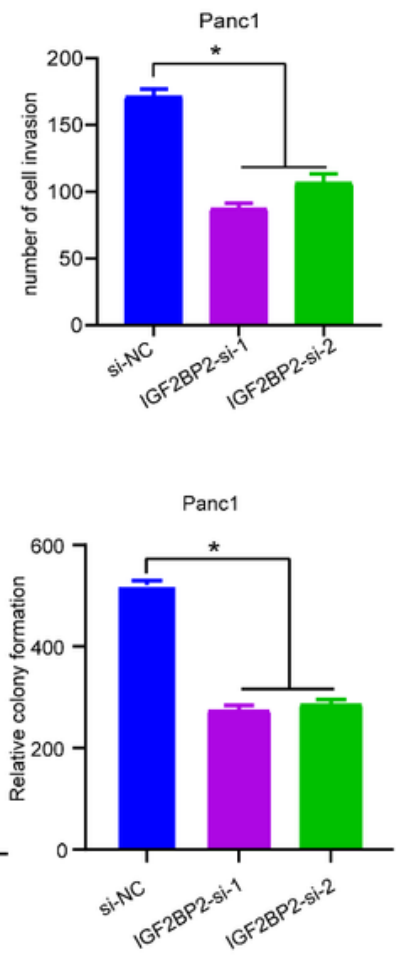

Merge
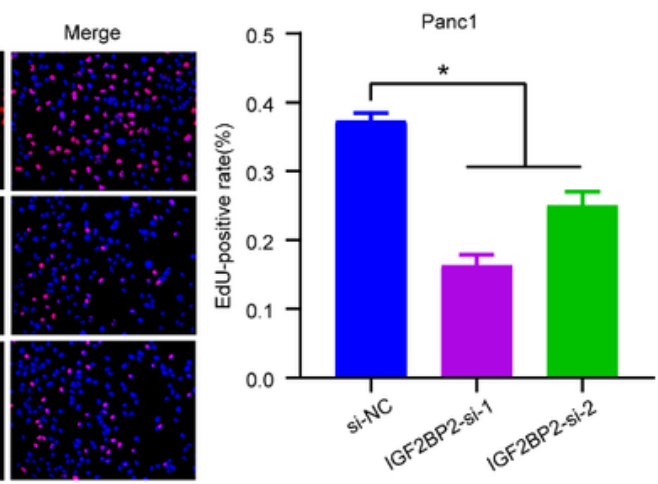

Figure 4

IGF2BP2 KO suppresses pancreatic carcinoma proliferation and migration ability. a-d CCK-8 and EdU assay revealed KO of IGF2BP2 inhibited pancreatic cell proliferation ability. e Transwell experiment revealed fewer cells migrated to the lower compartment in IGF2BP2 KO group compared with control group. f IGF2BP2 KO inhibited colony formation ability in PC cells. 
a

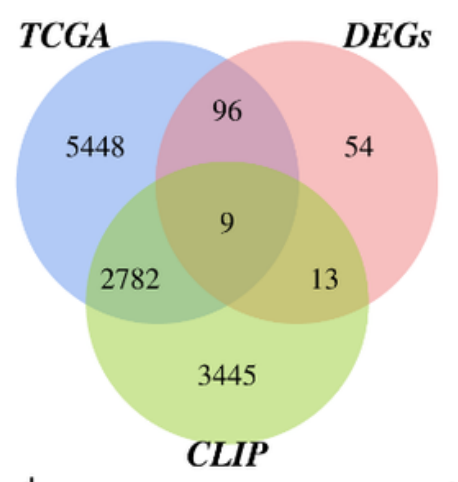

d
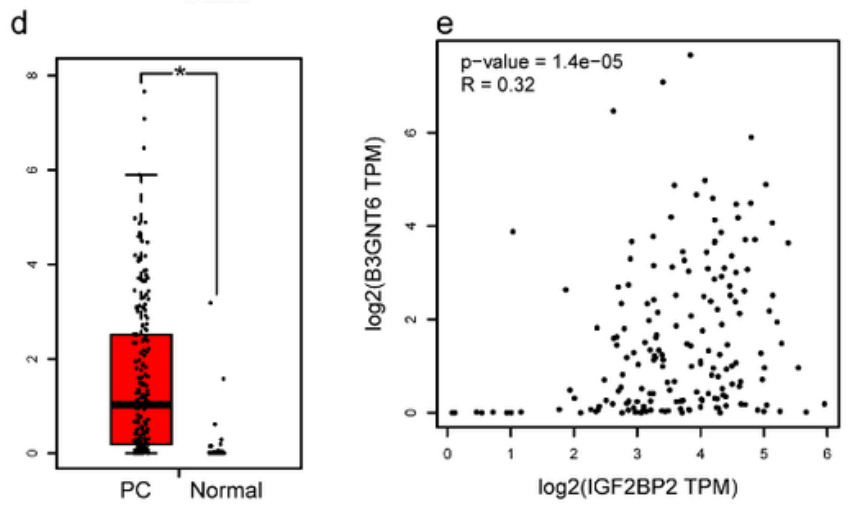

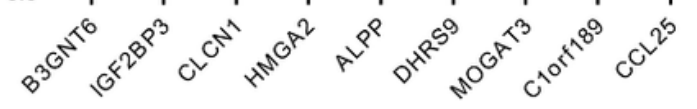

$f$
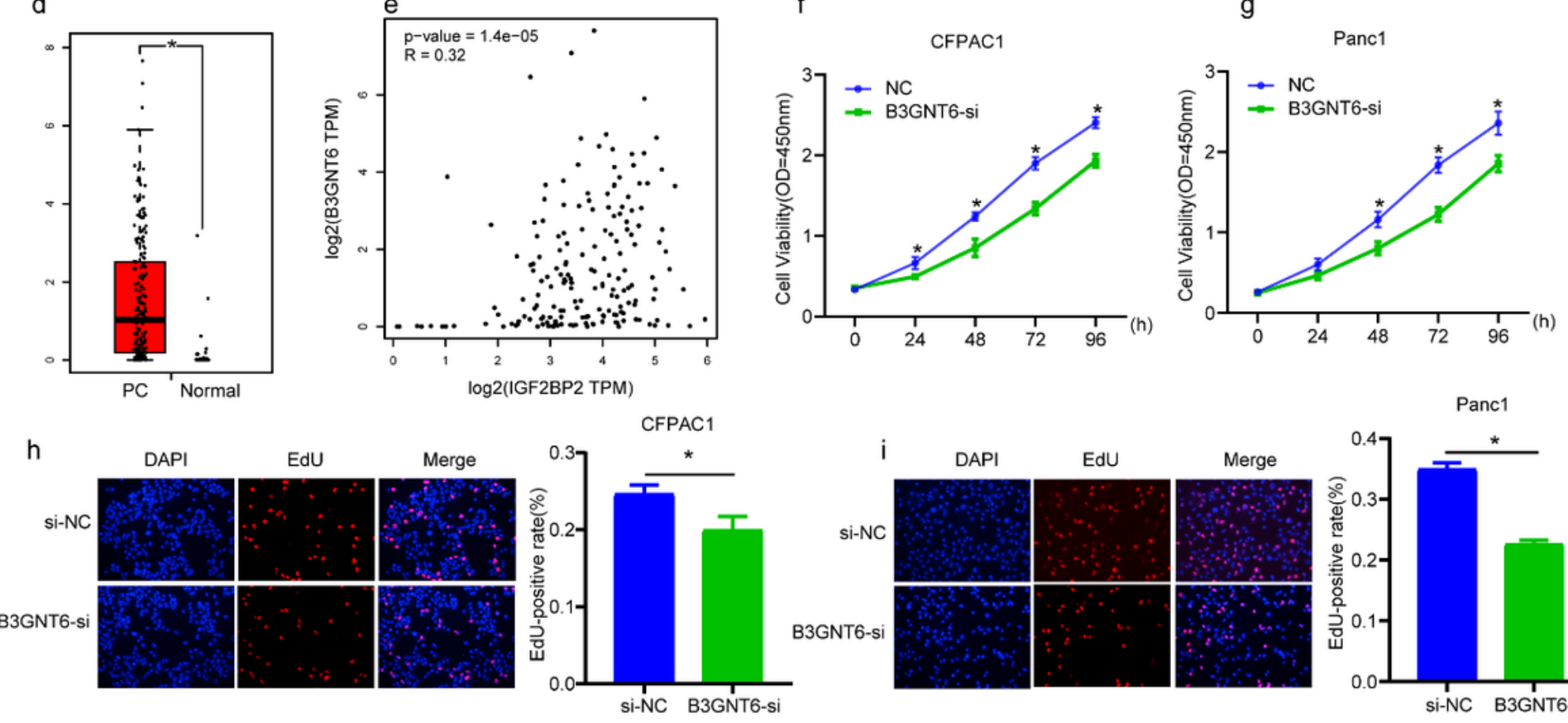

CFPAC1
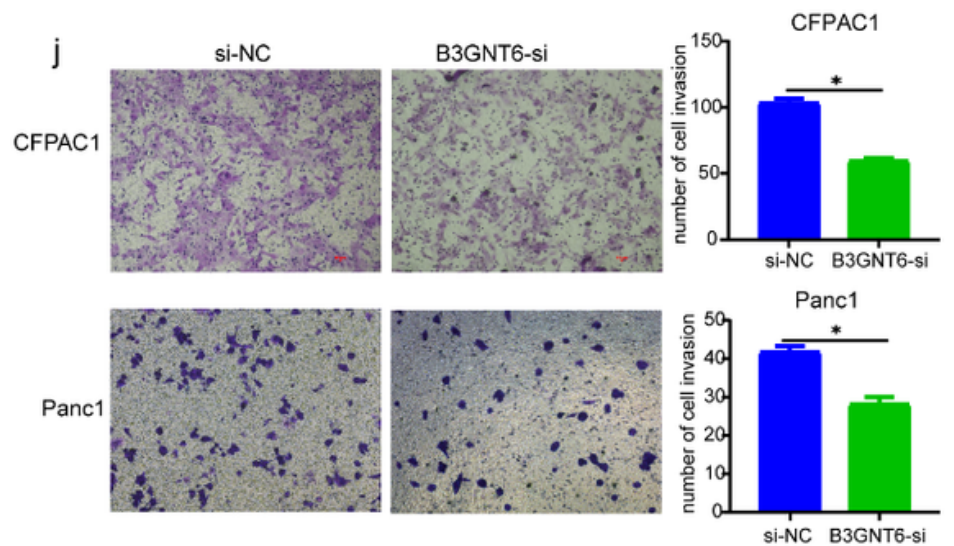
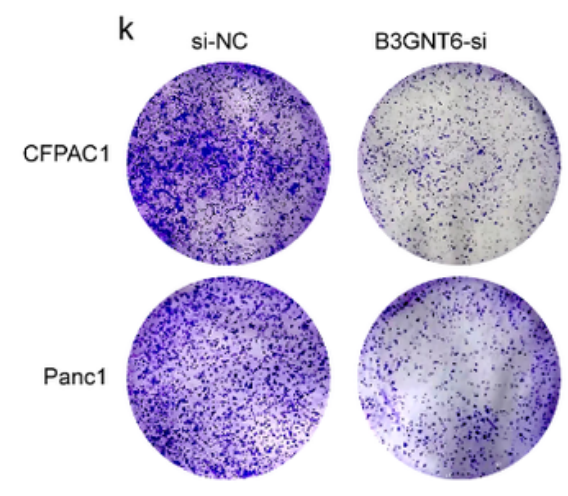
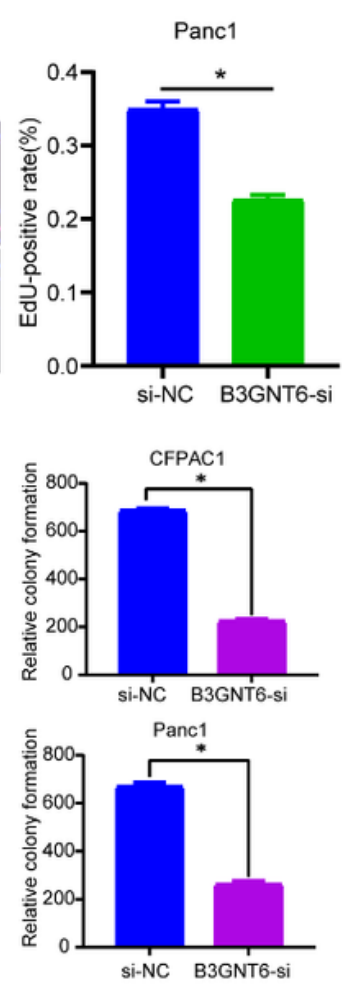

\section{Figure 5}

IGF2BP2 regulates B3GNT6 mRNA expression to promote pancreatic carcinoma. a Prediction of IGF2BP2 target gene, Venn diagram shows substantial and significant overlap among TCGA-GTEx, IGF2BP2-CLIP and DEGs between PA1 and PA2 subgroups. b Expression of predicted genes after IGF2BP2 KO, c m6A IP and IGF2BP2 IP verified verify the binding efficacy of target genes. d TCGA-GTEx reveals the high expression of B3GNT6 in PC. e Spearman correlation analysis reveals positive co-expression of IGF2BP2 and B3GNT6. f-i CCK-8 and EdU assay revealed KO of B3GNT6 inhibited pancreatic cell proliferation 
ability. j Transwell experiment revealed fewer cells migrated to the lower compartment in B3GNT6 KO group compared with control group. k B3GNT6 KO inhibited colony formation ability in PC.
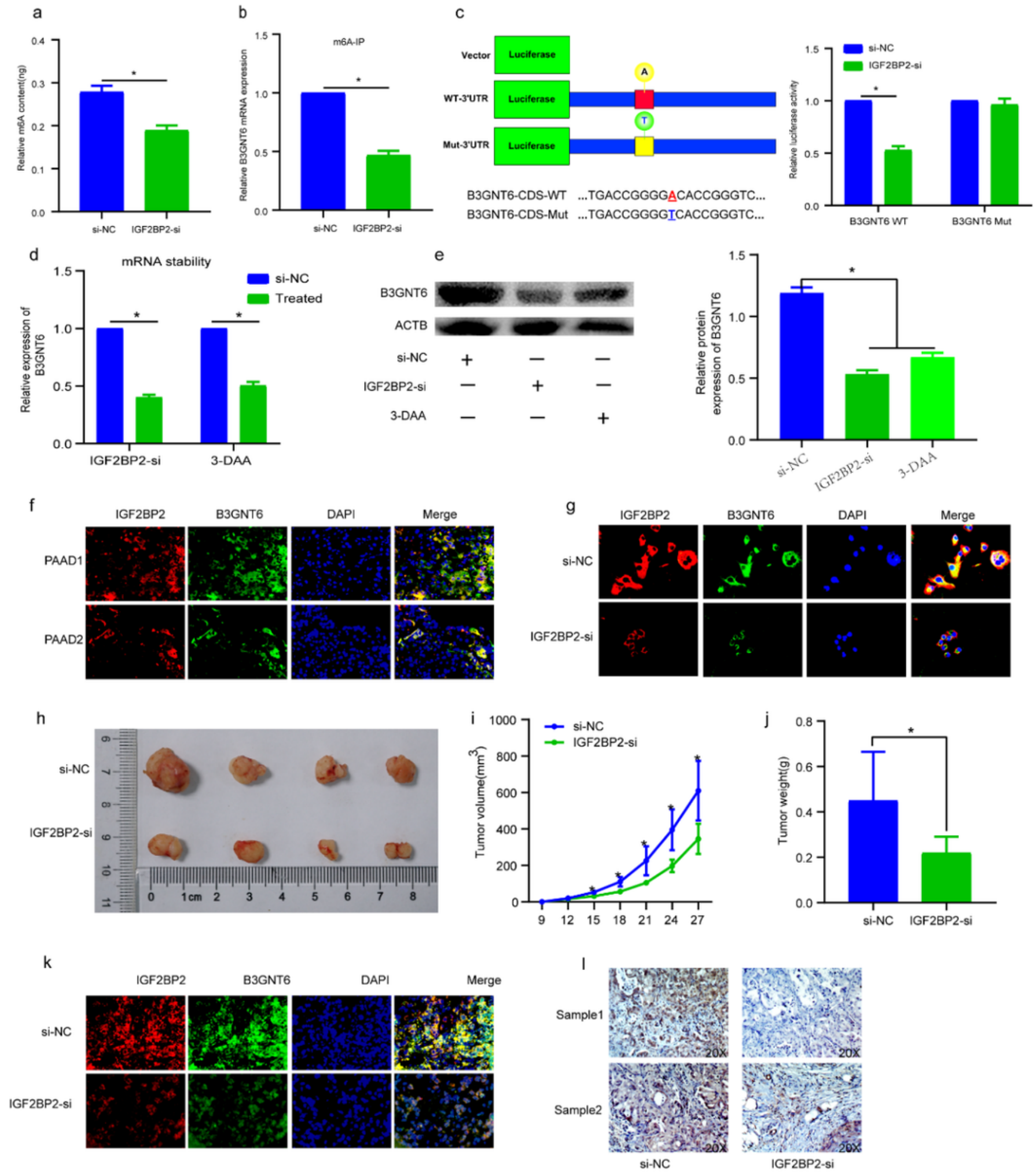

Figure 6

IGF2BP2 regulates B3GNT6 mRNA stability via an m6A-dependent manner and promotes m6A content in PC. a IGF2BP2 KO decreased m6A content in PC cells. b m6A IP verified IGF2BP2 KO inhibited B3GNT6 expression. c Dual luciferase reporter assays verified that B3GNT6 was observably methylated by 
IGF2BP2. d-e IGF2BP2 KO and 3-DAA decreased B3GNT6 mRNA stability and protein level. f-g Immunofluorescence staining confirmed the co-expression of IGF2BP2 and B3GNT6 in PC tissues (f) and cells (g). $\mathrm{h}$-j KO of IGF2BP2 effectively inhibited PC tumor growth in a nude mice model (h). The tumor size formed in the axillary implantation mice model was monitored every 3 days (i). Tumor weight was measured after mice was sacrificed, KO of IGF2BP2 drastically inhibited tumorigenicity in vivo (j). $\mathrm{k}$ Immunofluorescence staining confirmed the co-expression of IGF2BP2 and B3GNT6 in mice tumor tissue. I IHC(B3GNT6)-stained paraffin-embedded sections verified the low expression of B3GNT6 protein in IGF2BP2 KO group than control group.

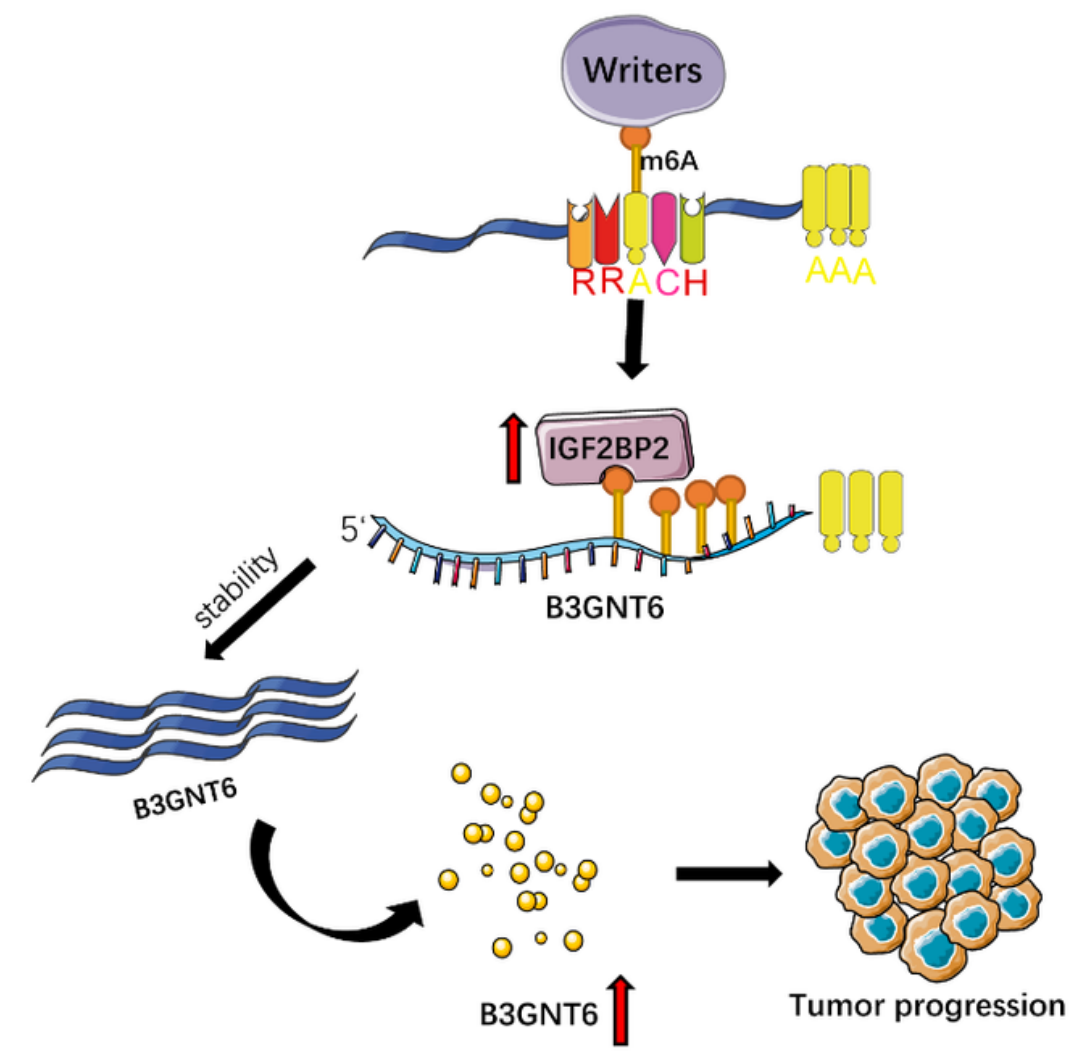

Figure 7

Mechanism diagram of IGF2BP2 promoting B3GNT6 expression via m6A methylation.

\section{Supplementary Files}

This is a list of supplementary files associated with this preprint. Click to download.

- AdditionalFig.docx

- AdditionalTable.docx 